\title{
然
}

\section{EL MODELO DE INNOVACIÓN Y EMPRENDIMIENTO EN SINGAPUR}

El objetivo del artículo es modelizar la estrategia que utiliza el Gobierno de Singapur para generar ecosistemas de innovación en distintos segmentos de forma acelerada, convirtiéndose en referencia en su región en pocos años. Se realiza un análisis sistemático de los cinco pilares de la innovación, de la idiosincrasia del país y de las políticas públicas aplicadas a cada uno de los ecosistemas generados, observándose la importancia que significa para Singapur estar en la vanguardia tecnológica y la atracción de talento. El resultado final es una modelización de las políticas públicas que utiliza el Gobierno singapurense para generar nuevos ecosistemas de innovación.

Palabras clave: innovación, startups, modelo, ecosistema, talento, tecnología, capital riesgo.

Clasificación JEL: G18, G28, O25, O31, O32, O38.

\section{Introducción}

Singapur es un país con una idiosincrasia muy particular, tanto por su condición de ciudad-Estado como por encontrarse entre el mundo occidental y el oriental, además de su posición geoestratégica como hub del comercio internacional. Gracias a su ubicación clave para el comercio, también se ha convertido en un centro financiero de tremenda relevancia en el Sudeste Asiático, llamada en ocasiones la «Suiza asiática» (Writer, 2020).

Pero Singapur, excolonia británica, tiene apenas 55 años de historia, en los que ha conseguido transformar unos terrenos pantanosos

* Oficina Económica y Comercial de la Embajada de España en Singapur.

Versión de enero de 2021.

DOI: https:/doi.org/10.32796/bice.2021.3132.7152 en una urbe moderna y de referencia en todo el mundo, en gran parte gracias a un gran intervencionismo político y a una clase política que ha sabido sacar partido de las pocas ventajas competitivas que disponía en cada momento histórico. Por ello, el People's Action Party (PAP), el partido que gobierna Singapur de forma ininterrumpida desde su fundación, goza de una gran confianza por parte de ciudadanos y empresas, que obedecen ciegamente sus indicaciones y recomendaciones, como se ha visto especialmente durante esta pandemia (Mas, 2020).

Gracias a este elevado nivel de influencia en el sector privado, el Gobierno ha logrado transformar la economía de Singapur a lo largo de su historia y pretende seguir haciéndolo. Las grandes economías mundiales como EE UU, China, Alemania o Japón luchan por $\triangleright$ 
mantenerse a la cabeza de la innovación y crear las grandes corporaciones que lideren el mundo, como han hecho Google, Apple, Facebook y, más recientemente, empresas como Huawei. Singapur, como Israel, identificó hace tiempo el capital humano como su recurso más valioso, combinado con la fuerte presencia de multinacionales extranjeras en la ciudad-Estado, y ha decidido apostar también por convertirse en un centro de referencia en innovación, objetivo que seguirá fortaleciendo en el seno de la crisis COVID-19 al haber aprobado recientemente un plan de inversión al alza en I+D para el periodo 2020-2025 de 25.000 millones de SGD (dólares de Singapur, unos 16.500 millones de euros), tratando de mantenerse en la senda del 1,8-2\% del producto interior bruto (PIB) que ha consolidado en los últimos años.

Para una población similar a la Comunidad de Madrid y un territorio como la isla de Menorca, presenta unos números incontestables, según su agencia pública de desarrollo económico EDB:

- 55.000 startups tecnológicas (empresas tecnológicas de nueva creación).

- 525 inversores establecidos.

- 216 aceleradoras.

- Aproximadamente 5.000 millones de SGD de inversión anuales.

A continuación, analizaremos cómo ha logrado aprovechar o crear los elementos esenciales que se requieren para generar un ecosistema de innovación.

\section{Financiación}

Iniciamos el análisis partiendo desde la financiación, porque puede actuar tanto de punto de partida para introducir a Singapur como de hilo conductor para presentar el resto de elementos de su ecosistema de innovación.

\subsection{Fondos soberanos}

A lo largo de su historia, la clase política de Singapur se ha caracterizado por una gran visión estratégica a largo plazo y por identificar y explotar las ventajas competitivas del país. Desde su fundación en los años sesenta, con una renta per cápita inferior a 1.500 USD (dólares estadounidenses), ha logrado, en apenas 55 años, transformarse en una de las urbes más modernas y desarrolladas del mundo. Una de las grandes ventajas competitivas de Singapur es la gran habilidad y capacidad de los gestores públicos, y, gracias a años de excelentes inversiones, actualmente Singapur dispone de dos de los fondos soberanos más importantes del mundo: Temasek y GIC (Elson, 2008), siendo el primero el encargado de las participaciones en las empresas singapurenses y el segundo el gestor de la rentabilidad de las reservas nacionales. Ambos fondos invierten, por tanto, en el extranjero y en el ecosistema tecnológico nacional.

Pero la importancia de estos fondos soberanos no se limita exclusivamente a la multiplicación de los fondos públicos, el acceso a financiación o la mejora de la rentabilidad de las aportaciones de los ciudadanos. Desde hace años, Singapur utiliza estos fondos como un elemento más de atracción de empresas e inversores al país y para tener influencia sobre el desarrollo del sector privado.

Estos dos fondos, que operan de forma más o menos transparente y con distintos instrumentos financieros según cada operación, además de realizar inversiones buscando rentabilidad, también realizan inversiones $\triangleright$ 
TABLA 1

COMPARATIVA DE LOS MEJORES ECOSISTEMAS DE INNOVACIÓN DEL MUNDO

\begin{tabular}{|c|c|c|c|c|c|c|c|c|c|c|c|}
\hline Métricas & $\frac{0}{\bar{O}} \frac{\bar{\sigma}}{\bar{\sigma}}$ & 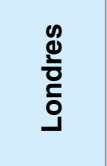 &  & $\frac{5}{d}$ & 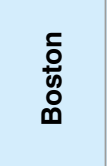 & : $\frac{8}{\frac{8}{9}}$ & 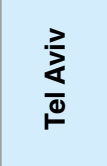 & $\begin{array}{l}: \bar{\pi} \\
\frac{5}{5} \\
\frac{5}{\pi} \\
\frac{\pi}{\omega}\end{array}$ & 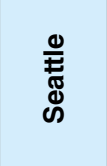 & 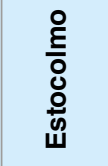 & $\begin{array}{l}\frac{1}{3} \\
\frac{2}{5} \\
\frac{5}{5} \\
\text { क }\end{array}$ \\
\hline Ranking & 1 & 2 & 3 & 4 & 5 & 6 & 7 & 8 & 9 & 10 & 17 \\
\hline $\begin{array}{l}\text { N.. } \text { de salidas }>\$ 50 M \\
(2017-2 T 2019)\end{array}$ & 142 & 44 & 65 & 27 & 51 & 29 & 27 & 20 & 18 & 16 & 5 \\
\hline $\begin{array}{l}\text { N.o de salidas }>\$ 1 \mathrm{~B} \\
(2017-2 \mathrm{~T} 2019)\end{array}$ & 25 & 3 & 5 & 16 & 7 & 3 & - & 6 & 3 & 2 & 2 \\
\hline $\begin{array}{l}\text { Valor del ecosistema (miles de } \\
\text { millones de USD) 2017-2T2019 }\end{array}$ & 676 & 92 & 147 & 344 & 96 & 120 & 47 & 138 & 30 & 44 & 21 \\
\hline Series B-to-A (2017-2T2019) ........... & 9 & 7 & 8 & 9 & 9 & 9 & 8 & 10 & 7 & 4 & 7 \\
\hline Series C-to-A (2017-2T2019) ........... & 9 & 6 & 7 & 10 & 8 & 7 & 8 & 10 & 7 & 6 & 8 \\
\hline $\begin{array}{l}\text { N. } . \text { Financiación inicial o } \\
\text { Early-Stage (2017-2T2019) }\end{array}$ & 5.007 & 3.051 & 2.926 & 856 & 918 & 1.478 & 938 & 508 & 534 & 575 & 848 \\
\hline $\begin{array}{l}\text { Financiación inicial o Early-Stage } \\
\text { (millones de USD, 2017-2T2019) ... }\end{array}$ & 18.129 & 6.015 & 8.316 & 7.480 & 3.898 & 3.597 & 3.337 & 8.722 & 1.546 & 944 & 1.446 \\
\hline $\begin{array}{l}N .{ }^{\circ} \text { de inversores de capital } \\
\text { riesgo (1T 2020) }\end{array}$ & 455 & 149 & 216 & 81 & 90 & 88 & 55 & 58 & 29 & 24 & 38 \\
\hline $\begin{array}{l}\text { Años de experiencia media de los } \\
\text { VC del ecosistema (1T 2020) }\end{array}$ & 11 & 10 & 9 & 11 & 14 & 10 & 12 & 8 & 11 & 16 & 14 \\
\hline $\begin{array}{l}\text { Ratio de inversores por encima } \\
\text { del ratio medio de salida } \\
\text { (1T 2020) }\end{array}$ & $18,5 \%$ & $8,7 \%$ & $8,8 \%$ & $6,2 \%$ & $20,0 \%$ & $10,2 \%$ & $0,0 \%$ & $3,4 \%$ & $10,3 \%$ & $8,3 \%$ & $10,5 \%$ \\
\hline $\begin{array}{l}\text { Nuevos inversores activos entre } \\
\text { inversores totales activos } \\
\text { (1T 2020) }\end{array}$ & $34,5 \%$ & $33,6 \%$ & $34,3 \%$ & $16,0 \%$ & $25,6 \%$ & $44,3 \%$ & $32,7 \%$ & $22,4 \%$ & $37,9 \%$ & $33,3 \%$ & $23,7 \%$ \\
\hline $\begin{array}{l}\text { N. de inversores que han sido } \\
\text { activos } 5 \text { años o menos } \\
\text { (1T 2020) }\end{array}$ & 157 & 50 & 74 & 13 & 23 & 39 & 18 & 13 & 11 & 8 & 9 \\
\hline $\begin{array}{l}\mathrm{N} . . \text { o de salidas por encima de } 50 \\
\text { millones de USD } 2009-2018\end{array}$ & 511 & 145 & 143 & 89 & 113 & 92 & 89 & 49 & 41 & 30 & 11 \\
\hline $\begin{array}{l}\text { N.ㅇ de salidas por encima } \\
\text { de } 1.000 \text { millones de USD } \\
2009-2018\end{array}$ & 57 & 7 & 9 & 25 & 12 & 8 & 4 & 8 & 5 & 4 & 2 \\
\hline $\begin{array}{l}\text { N. }- \text { de rondas de financiación de } \\
\text { Series A en 2009-2018 }\end{array}$ & 3.841 & 1.136 & 1.792 & 1.034 & 890 & 867 & 748 & 549 & 459 & 379 & 352 \\
\hline
\end{tabular}

estratégicas para controlar grandes multinacionales o para desarrollar industrias de interés para Singapur.

Un ejemplo de ello es OLAM International, controlada al $54 \%$ por Temasek. Singapur importa el $90 \%$ de sus alimentos y apenas tiene producción agrícola debido a su pequeño tamaño y terreno arable. Controlar OLAM permite a Singapur un mayor control sobre la provisión de productos agroalimentarios desde otros países de la zona e incluso de Europa, así como involucrarse en su proceso de internacionalización; a su vez, OLAM está invirtiendo en innovación en el sector agrifoodtech, justamente un sector crítico para Singapur y por el que el Gobierno singapurense está apostando fuerte. Que OLAM, una de las mayores multinacionales del sector agroalimentario, invierta en agrifoodtech, empuja al sector a sumarse a la tendencia y ayuda a Singapur a convertirse $D$ 
en un centro agrifoodtech de referencia en el mundo (Whitehouse, 12 de febrero de 2020).

Este tipo de "campeones nacionales», grandes empresas sobre las que el Gobierno de Singapur tiene influencia, ayuda a la clase política a orientar el desarrollo de cada sector de acuerdo con los intereses estratégicos del país.

\subsection{Sector financiero}

En primer lugar, como se ha mencionado anteriormente, Singapur es una de las plazas financieras más importantes de Asia, por lo que los ecosistemas de innovación tienen un gran número de potenciales inversores en el país, una ventaja indudable y un requisito necesario para la creación de un ecosistema de innovación activo.

El impacto de los fondos soberanos en el ecosistema de innovación es indirecto en la mayoría de los casos, ya que rara vez realizan inversiones directamente en startups. Sin embargo, de la misma forma que actúan a través de multinacionales como OLAM, estos fondos también han desarrollado instrumentos y herramientas financieras para ayudar a desarrollar el sector del capital riesgo en Singapur.

Es habitual que en Singapur muchos fondos de VC (capital riesgo, siglas del término en inglés, venture capital) o de PE (capital inversión, siglas del término inglés private equity) cuenten con dinero de fondos públicos para realizar sus inversiones en el ecosistema de innovación. Es frecuente que Temasek y GIC, además de realizar inversiones más o menos directas a través de sus ramas de VC, que en el caso de Temasek son Vertex Holdings y Heliconia Capital Management, participen en fondos VC en Singapur o en el extranjero (Yap, 2020), con el objetivo, en algunos casos, de que no se identifique de manera inmediata o muy directa la participación de Singapur como país en dichas inversiones, muchas veces estratégicas.

Esta facilidad de acceso a inversores públicos para los fondos VC en Singapur provoca que las startups tengan grandes facilidades para encontrar financiación y que los inversores privados vean disminuir su riesgo, ya que las inversiones de este tipo de fondos cuentan más adelante con apoyos públicos. Además, el sector público en Singapur dispone de muchos programas dedicados al asesoramiento e incubación de startups que ayudan a emprendedores a realizar estas conexiones con inversores a través de aceleradoras y programas de mentoring para emprendedores, como veremos más adelante.

\section{Academia}

Una de las principales ventajas competitivas de Singapur, y una de las razones por la que la clase política singapurense ha identificado la innovación como una oportunidad estratégica para el país, es la excelente formación y preparación de la mano de obra en el país o, al menos, las excelentes capacidades del sistema educativo de Singapur, valorado, en la mayoría de foros, como uno de los mejores del mundo. Las universidades de Singapur están habitualmente presentes en posiciones destacadas en los rankings internacionales de mayor prestigio (QS Quacquarelli Symonds Limited 1994, 2020).

Como ejemplo, la National University of Singapore (NUS), la principal universidad pública del país, ocupa actualmente el undécimo lugar en el QS World University Rankings, y el primero en el QS Asia University Rankings 2021. La escuela ocupa el séptimo lugar en el mundo $D$ 
en el área de ingeniería y tecnología, y el sexto en ingeniería civil. También está entre las diez primeras en ciencias sociales y gestión.

La Universidad Tecnológica de Nanyang (NTU) ha sido clasificada solo dos puestos por detrás de la NUS en el QS World University Rankings, ocupando el puesto 13 del mundo y el tercero de Asia. Está muy bien considerada por sus cursos de ingeniería, figura entre las cinco primeras del mundo en el amplio campo de la ingeniería y la tecnología y ocupa el tercer puesto en ciencias de los materiales.

Sin embargo, el mayor éxito del sistema educativo singapurense, en relación con la innovación, radica en la creación de programas y entes dedicados a conectar la investigación pura en las universidades con el mundo empresarial y las empresas. Es habitual en los sectores innovadores en auge que las universidades cierren acuerdos de colaboración con multinacionales para establecer programas que ayuden a llevar la innovación del laboratorio al mercado.

Como ejemplo, en el caso de la NUS, se ha creado NUS Enterprise, un ente independiente bajo el paraguas de la universidad que se dedica a enfocar los programas de investigación a la comercialización y su salida al mercado. Disponen de instalaciones, fondos y un amplio abanico de programas de incubación, aceleración y fondos para crear startups a partir de los programas de investigación e impulsarlas y conectarlas en sus fases más iniciales. Es más, habitualmente colaboran con grandes empresas para la creación de programas especializados. De hecho, algunos de los centros de referencia del panorama de innovación en Singapur forman parte de NUS, como Pier71, ICE71, Singapore Airlines Accelerator Program, DSTA@71 o Tencent-NUS Cloud Startup Program, entre otros. Algunas de ellas se dedican a acelerar o incubar startups de un sector concreto, como
ICE71 en el sector de la ciberseguridad o Pier71 en el sector marítimo, y otras son el resultado, una vez más, de colaboraciones público-privadas, como el caso de Singapore Airlines, o colaboraciones entre entes públicos, como el caso de DSTA, ente público dedicado a la adquisición de tecnología militar.

El Centro de Tecnologías Cuánticas de Singapur es otro ejemplo de su apuesta por lo más vanguardista en términos tecnológicos. Este centro de investigación de excelencia se encuentra en la NUS y está auspiciado por la propia Oficina del Primer Ministro a través de la National Research Foundation (NRF), reuniendo bajo la batuta de un director español a físicos, informáticos e ingenieros para realizar investigaciones básicas sobre física cuántica y construir dispositivos basados en fenómenos cuánticos.

Es destacable también la función de IPI Singapore como catalizador de la innovación. IPI Singapore, subsidiaria de ESG, es un ente público que tiene como misión actuar como intermediario entre el tejido empresarial y los investigadores y otros agentes innovadores. Además de servicios relacionados con la innovación abierta, actúa como un marketplace (mercado) de innovación en el que se da publicidad a patentes innovadoras en busca de empresas que lleven la innovación al mercado y en el que se ayuda a empresas a encontrar soluciones innovadoras. De nuevo, la clave del éxito de Singapur en relación con la innovación es su capacidad de generar investigación e innovación orientada al mercado.

\section{Demanda}

El Gobierno de Singapur identificó desde sus inicios la necesidad de atraer empresas para generar crecimiento económico en el $\triangleright$ 
país. Por esto, la atracción de inversiones es una de las actividades clave para el crecimiento del país y disponen de programas y entes públicos dedicados únicamente a ello. Una parte importante de la propia idiosincrasia del país existe a raíz de la necesidad de ser atractivo para la actividad empresarial. Con bajos tipos impositivos en un contexto de estabilidad política, su conectividad debida a su gran ubicación geográfica, punto de paso obligado para la mayoría del tráfico de mercancías del mundo, una cultura a caballo entre Occidente y Oriente, rechazo cerrado a la corrupción y apuesta por el imperio de la ley, y una población políglota, multicultural y con un elevado nivel de preparación y formación, todo apunta a que sea el lugar ideal para el establecimiento de grandes empresas.

Como resultado, en Singapur se encuentra la gran mayoría de multinacionales, ya sea en forma de sede, de centro de I+D o de sede regional para Asia. Existen 7.000 grandes empresas establecidas en Singapur, 3.500 de ellas con sede regional para Asia.

La presencia de grandes empresas en el país, gracias a los programas públicos de atracción de inversiones, y de los «campeones nacionales», grandes empresas controladas por fondos soberanos, genera un ecosistema empresarial muy ligado al sector público, en el que las compañías acostumbran a apostar por lo que sugiere el Gobierno, y permite, mediante una mezcla de incentivos fiscales y no fiscales, generar rápidamente tendencias en el mercado para impulsar la innovación en el sentido que busca el Gobierno.

Como resultado de ello, un gran número de multinacionales en Singapur ha establecido programas de innovación abierta y aceleradoras privadas en colaboración con entidades públicas. Aunque se han convertido en algo común en todos los sectores de la innovación, el sector fintech, más maduro que el resto, destaca especialmente con aceleradoras privadas de Mastercard, OCBC, DBS, UOB, o PayPal, entre otros. Durante 2020 se han presentado 42 Open Innovation Challenges, esencialmente programas de innovación abierta en el que se plantean problemas a solucionar por las grandes empresas, y la startup que proponga la mejor solución logra financiación para desarrollarla y un gran cliente inmediato.

Por otra parte, Singapur actúa también como centro de negocios en dos sentidos. En primer lugar, es el centro de negocios de referencia para el Sudeste Asiático, por lo que todas aquellas grandes empresas con interés en dicha región tendrían sede en Singapur. En segundo lugar, como hemos mencionado ya, Singapur es uno de los nexos de unión entre Asia y Occidente, de forma similar a Hong Kong, por sus nexos de unión con China y el Sudeste Asiático y su condición de antigua colonia británica. Por ello, muchas grandes empresas están presentes en Singapur con centros de I+D y sedes regionales, lo que genera que muchos centros de decisión para la zona asiática se encuentren en Singapur. Cuando los centros de decisión y los centros de investigación se encuentran en Singapur, es natural que la demanda de innovación crezca en el mercado.

\section{Oferta}

La oferta es quizá el elemento más complicado de generar de forma acelerada para Singapur. Pese a que dispone de un capital humano con elevados niveles de formación y programas de investigación exitosos, en términos generales la población de Singapur no es muy emprendedora y es limitada en número. Por ejemplo, en el Global Entrepreneurship $\triangleright$ 
Monitor, los datos de 2013 y 2014 muestran una intención muy baja de emprender, de menos de la mitad en comparación con la media mundial (Global Entrepreneurship Research Association, 2014).

Esto es debido a un nivel de vida elevado sin necesidad de arriesgar el futuro profesional y las finanzas personales y/o familiares, y a la cultura asiática de aversión al riesgo, en el que las familias emplazan a sus jóvenes a estudiar y encontrar un trabajo estable en una gran empresa $(\mathrm{Ng}, 2020)$, sin olvidar la meritocracia que se aplica en una Administración pública singapurense muy bien considerada.

Singapur no ceja en su empeño de generar, incluso artificialmente, nuevas startups a nivel local que afronten soluciones a los problemas más disruptivos. En el campo de la robótica, por ejemplo, el Advanced Remanufacturing and Technological Center ARTC es una colaboración público-privada entre la agencia nacional de innovación A*STAR, NTU y un buen número de multinacionales industriales, que busca ser una plataforma generadora de nuevos modelos de desarrollo de la industria 4.0 que satisfaga los requerimientos particulares en este campo de grandes empresas a través de una combinación de tecnología, talento y emprendimiento local, y también extranjero.

Sin embargo, y aunque las nuevas generaciones están invirtiendo la tendencia, gracias a la normalización de la innovación en Singapur, al elevado crecimiento de las startups en este mercado, al fortalecimiento de Singapur como hub tecnológico además de comercial y al amplio apoyo público a los emprendedores, además de la inestabilidad económica provocada por la crisis de la COVID-19, los emprendedores en Singapur son escasos e insuficientes para los planes del país, por lo que el Gobierno ha puesto en marcha todo tipo de programas para atraer a los futuros gigantes tecnológicos. Como ya han hecho desde su fundación, los singapurenses son grandes expertos en la atracción de empresas y tan solo han tenido que cambiar el objetivo de las grandes empresas a las startups internacionales más prometedoras.

Enterprise Singapore, la agencia pública encargada del desarrollo empresarial de Singapur, lidera la promoción como centro de innovación mundial y, para ello, además de incentivar y promocionar la creación e internacionalización de startups, también dispone de iniciativas como la GIA (Global Innovation Alliance), una red de centros de innovación en todo el mundo dedicados a la colaboración internacional en innovación, que utilizan para identificar startups y tecnologías prometedoras, a las que ofrecen distintos programas para ayudar en su aterrizaje en Singapur.

Mediante esta actividad de atracción de empresas innovadoras, Singapur consigue convertirse en referencia para la innovación en Asia, lo cual retroalimenta y facilita su actividad de atracción de startups y genera la masa crítica necesaria para impulsar la creación de unas propias. Una vez se genera dicha masa crítica, es muy fácil para Singapur atraer y absorber startups de interés para el ecosistema.

Como ejemplo de ello, una de las empresas de inteligencia artificial más importantes de Singapur es Taiger. Taiger es una empresa dedicada al procesamiento de documentación e información mediante inteligencia artificial. Creada en España en 2010, y tras años de actividad en España y EE UU, desde Singapur identificaron el potencial de la empresa y facilitaron su aterrizaje en el país, desde donde se ha convertido en una referencia, con clientes en el sector bancario y en el sector público (Lucena, 9 de abril de 2018). 


\section{Apoyo público}

Por último, aunque el apoyo público está presente en todos los elementos presentados anteriormente, es sorprendente el gran número de programas, entidades y recursos enfocados al desarrollo de Singapur como centro de innovación mundial. Analizar pormenorizadamente todos los programas sería demasiado extenso para el artículo que nos ocupa, pero es destacable que las iniciativas estratégicas nacionales están orientadas a la innovación.

En primer lugar, Singapur desde hace años se ha planteado convertirse en una smart nation. Partiendo del concepto smart cities, centrado en la innovación, en la gestión de los espacios y servicios de una ciudad pero con la visión y el empuje presupuestario de Singapur como ciudad-Estado, se ha propuesto como objetivo digitalizar y automatizar tantos procesos como sea posible. Para ello existe una agencia pública, la IMDA (Infocomm Media Development Authority), dedicada a la digitalización de la economía de Singapur. Singapur es desde hace años una de las referencias en los rankings internacionales de digitalización (IMDA, 2020), lo cual favorece la importancia de nuevas tecnologías transversales innovadoras como la inteligencia artificial, internet de las cosas, $5 \mathrm{G}$ o tecnologías en la nube, entre muchos otros.

Por otra parte, Singapur también se ha propuesto como objetivo nacional generar el $30 \%$ de sus necesidades nutricionales, desde el $10 \%$ actual, a través de la innovación en agrifoodtech. Desde hace años, Singapur ha identificado su dependencia de terceros para la alimentación de su población y ha empleado multitud de estratagemas para asegurarse el suministro de alimentos, algo que ha logrado con algunas dificultades incluso con la crisis de la COVID-19. Sin embargo, esta crisis ha acentuado la importancia de esta debilidad estratégica y ha decidido apostar por la innovación para mitigarla.

Estos dos ejemplos dan una idea de la importancia estratégica de la innovación para Singapur como Estado y la magnitud de la apuesta del Gobierno singapurense al completo por la innovación. Otros ejemplos de sectores actualmente estratégicos para Singapur podrían ser el sector de la ciberseguridad, el logístico o la sanidad.

Al tratarse de iniciativas nacionales, el apoyo público se canaliza a través de todas las agencias públicas con una aproximación Whole-of-Government (WGA), en el que todas las agencias trabajan estrechamente para encontrar soluciones de forma conjunta. Sin embargo, la agencia que ejerce de punta de lanza, como se ha mencionado anteriormente, es Enterprise Singapore, que se coordina con otras agencias como EDB, responsable de reforzar la posición de Singapur como centro global de talento y negocios a través de la atracción de empresas; IMDA, agencia responsable del desarrollo de las telecomunicaciones y digitalización de Singapur; o A*STAR, la agencia encargada de potenciar la investigación en Singapur y su comercialización.

Originalmente, Enterprise Singapore (conocido como ESG) tenía un enfoque más orientado a la internacionalización del tejido empresarial singapurense, pero, desde el año 2018, absorbe la agencia SPRING, la encargada hasta entonces de dar apoyo público a la innovación, y reenfoca su misión hacia el desarrollo competitivo del tejido empresarial de Singapur, lo que conlleva el desarrollo internacional de su ecosistema de innovación. Lo hace fomentando la creación y llegada de startups, favoreciendo la aproximación de corporates a Singapur y uniendo ecosistemas internacionales $\triangle$ 
con el singapurense a través de la Global Innovation Alliance.

A raíz de este cambio de orientación se crea una división dentro de ESG conocida como Startup SG, que fue creada para representar los intereses compartidos de la comunidad de startups y unifica los esfuerzos para apoyar el ecosistema en el marco de sus diversas iniciativas y programas. Con Startup SG, las startups y los asociados del ecosistema pueden descubrir y acceder más fácilmente a las vías de apoyo disponibles además de tener sus propios instrumentos. En 2018 se puso en marcha también la startup SG Network con el fin de acercar aún más el ecosistema de startups tecnológicas de Singapur y fomentar la proliferación de asociaciones innovadoras y de colaboración. La plataforma, que es un ecosistema virtual de entidades de la comunidad de startups tecnológicas de Singapur, permite que las locales se perfilen y se pongan en el radar de los actores del ecosistema, ampliando sus oportunidades de crecimiento. Es decir, es un directorio del ecosistema startup en Singapur que da visibilidad a todos los actores a nivel nacional e internacional. De la misma forma, es necesario estar registrado en Startup SG para solicitar programas de ayuda relacionados con el emprendimiento tecnológico. Las ayudas ofrecidas son tanto programas de aceleración e incubación como de apoyo financiero y programas de desarrollo y captación de talento.

Finalmente, ESG incluso dispone de un fondo propio denominado SEEDs que le permite coinvertir con otras entidades en startups singapurenses de proyección.

Por último, el entorno legal y fiscal de Singapur, una vez más, se ha adaptado para convertir la ciudad-Estado en el lugar ideal para la creación y desarrollo de startups. Figuras como las recientemente creadas Variable Capital
Companies (VCC), copiadas de Hong Kong, figuras similares a las SICAV que permiten la inversión flexible en startups, visados de emprendimiento, exenciones y deducciones fiscales, entre muchas otras medidas, incentivan de forma indirecta la creación de startups en Singapur y convierten al país en un lugar atractivo para inversores de capital riesgo.

\section{Conclusiones}

Singapur, una vez más, ha actuado como una gran empresa, con un análisis objetivo de su situación, de sus fortalezas y debilidades, planteando un plan estratégico con una visión clara a largo plazo para mitigar sus debilidades y explotar sus fortalezas. Conociendo que sus fortalezas no radican en sus recursos naturales (Singapur no dispone de agua o minerales, entre otros recursos) o agrícolas (la aportación agrícola a día de hoy al PIB singapurense es muy residual debido principalmente a la falta de suelo y a su coste de oportunidad), o en la intensidad de la fuerza laboral (la población singapurense está estancada en torno a los cuatro millones de ciudadanos, siendo la población extranjera en Singapur la que incrementa o reduce el total de personas que habitan la ciudad-Estado, que cuenta con tasas de paro muy bajas), Singapur se ha volcado en línea con las teorías de crecimiento endógeno de Romer (1986) y Lucas (1988) en fomentar una productividad asociada al progreso técnico y al capital humano, a su capacidad de atracción de empresas y su papel de nexo entre Asia y Occidente, a su capacidad de inversión y al control del sistema financiero, siendo capaz de realizar un análisis de la situación de la economía mundial, identificando en la innovación una oportunidad única para un país como Singapur. $\quad \square$ 
Una vez identificada la oportunidad, han avanzado con una prueba piloto en el sector que más controlan: el sector financiero. Utilizando su influencia en los principales bancos del país y su control sobre el sistema financiero y descubriendo cómo podrían utilizar mejor sus fortalezas y recursos para generar los elementos necesarios para un ecosistema de innovación, lograron un gran éxito, convirtiendo Singapur en un referente en el sector fintech a nivel internacional. Actualmente, la feria fintech más importante del mundo, el Singapore Fintech Festival, tiene lugar en Singapur de forma anual.

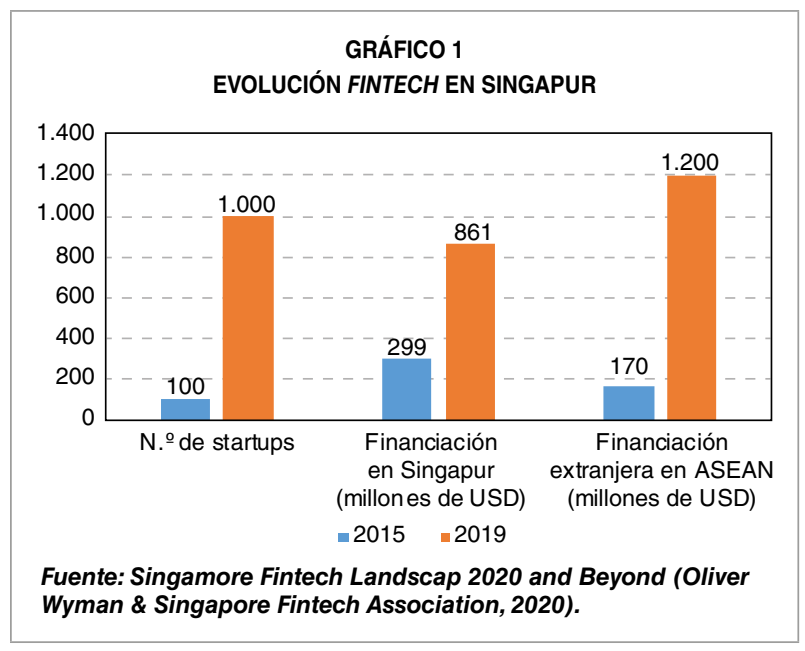

Tras el éxito logrado con el sector fintech, Singapur ha hecho esfuerzos focalizados en aquellos sectores donde tiene una mayor influencia y hay presencia de multinacionales. Por ejemplo, hace unos años apostaron muy fuertemente por el sector medtech, ya que muchas grandes empresas de tecnología médica tienen presencia en Singapur y lograron un éxito relativo, aunque lejos del sector financiero. La actual crisis COVID-19, sin duda, ampliará la metodología a todo el sector healthtech.

Más recientemente, han decidido aplicar este modelo a otros sectores que permitan fortalecer su posición estratégica como centro de negocios para Asia y mitigar sus debilidades. Tal y como se ha explicado, un ejemplo de ello es la gran apuesta reciente por el sector agrifoodtech, debido a la presencia en Singapur de grandes multinacionales del sector agroalimentario y «campeones nacionales» con influencia para mitigar una de las grandes debilidades de Singapur: la dependencia de terceros para alimentar a su población. Otro ejemplo es el sector de la ciberseguridad. Singapur ha apostado muy fuertemente por la digitalización para reforzar su posición como centro de negocio para Asia, pero requiere de una fuerte industria de ciberseguridad para proteger la digitalización de sus negocios y del sector financiero, y la gran mayoría de grandes empresas de ciberseguridad ya tienen presencia en la ciudad.

Áreas más transversales como la inteligencia artificial y el internet de las cosas llevan tiempo siendo aspectos sobre los que Singapur aplica un seguimiento especial y seguirán siendo determinantes a pesar de las reconsideraciones presupuestarias que la crisis actual ocasiona en todo el mundo.

A modo de resumen, el modelo de innovación de Singapur está basado en la influencia del sector público sobre el privado. El Gobierno, en primer lugar, identifica aquellos sectores estratégicos para el país y aquellos en los que tiene capacidad de influencia. Una vez identificados, anuncia su intención de invertir y apostar fuertemente por esos sectores y entra en contacto con las grandes empresas del sector para establecer colaboraciones público-privadas tanto con las universidades como con sus agencias públicas. A continuación, rastrea los mercados internacionales en busca de startups prometedoras de ese sector y negocia todo tipo de incentivos y ayudas para atraerlas y $\triangleright$ 
generar masa crítica, a la vez que apoya de forma directa, mediante financiación y acceso a grandes empresas y al sector público, a aquellas startups singapurenses más prometedoras. Finalmente, a través de eventos, premios y de la masa crítica generada, se reconoce la presencia en Singapur de un ecosistema de referencia para ese sector concreto en toda la región. Todo ello acompañado de medidas fiscales y legales para facilitar el emprendimiento y la innovación de forma genérica.

\section{Bibliografía}

Elson, A. (2008). The Sovereign Wealth Funds of Singapore. Ideas. https://ideas.repec.org/a/wej/ wldecn/343.html

Global Entrepreneurship Research Association (2014). Entrepreneurship in Singapore. https:// www.gemconsortium.org/economy-profiles/ singapore

IMD World Competitiveness Center (2019). IMD World Digital Competitiveness Ranking. https:// www.imd.org/globalassets/wcc/docs/release-2019/digital/imd-world-digital-competitiveness-rankings-2019.pdf

IMD World Competitiveness Center (2019). Smart City Index. https://www.imd.org/globalassets/wcc/ docs/smart_city/smart_city_index_digital.pdf

IMDA (2020). Singapore's Ranking in Infocomm. https://www.imda.gov.sg/infocomm-medialandscape/research-and-statistics/singaporesranking-in-infocomm

Lucas Jr, R. E. (1988). On the mechanics of economic development. Journal of Monetary Economics, 22(1), 3-42.

Lucena, B. (9 de abril de 2018). Taiger, el «tigre tecnológico" español que conquista Asia. $A B C$. https://www.abc.es/economia/abci-taiger-tigretecnologico-espanol-conquista-asia-201804080222 noticia.html
Mas, X. (2020). Singapur, el país 'niñera', impone su ley ante el virus. La Vanguardia. https://www. lavanguardia.com/vida/salud/20201212/ 6112588/singapur-pais-ninera-impone-ley.html

$\mathrm{Ng}$, A. (25 de septiembre de 2020). Singapore's entrepreneurs have often had to overcome family pressure - but that may be slowly changing. CNBC. https://www.cnbc.com/2020/09/25/family-pressure-on-singapore-entrepreneurs-eases-. html

Oliver Wyman Forum (2019). Global Cities Al Readiness Index. https://www.oliverwymanforum.com/ city-readiness/global-cities-ai-readiness-index-2019.html

QS Quacquarelli Symonds Limited 1994 (27 de 11 de 2020). Study in Singapore. Topuniversities. https://www.topuniversities.com/where-to-study/ asia/singapore/singapore/guide

Romer, P. M. (1986). Increasing returns and long-run growth. Journal of Political Economy, 94(5), 1002-1037.

Whitehouse, D. (12 de febrero de 2020). Olam may need to change where it lists to convince on reorganisation. TheAfricaReport. https://www.theafricareport.com/23360/olam-may-need-changewhere-it-lists-to-convince-on-reorganisation/

World Bank Group (2020). Doing Business 2020. http://documents1.worldbank.org/curated/ en/688761571934946384/pdf/Doing-Business2020-Comparing-Business-Regulation-in-190Economies.pdf

World Economic Forum (2019). The Global Competitiveness Report. http://www3.weforum.org/docs/ WEF_TheGlobalCompetitivenessReport2019. pdf

Writer, S. (2020). Switzerland of Asia: Singapore increases presence as tech hub. Nikkei Asia.

Yap, T. Y. (2020). Temasek injects US\$50M into India's new early-stage VC fund Info Edge Ventures. https://e27.co/temasek-injects-us50m-intoindias-new-early-stage-vc-fund-info-edge-ventures20201218/ 
